\title{
Persistent Nociception Triggered by Nerve Growth Factor (NGF) Is Mediated by TRPV1 and Oxidative Mechanisms
}

\author{
Michael A. Eskander, ${ }^{1}$ Shivani Ruparel, ${ }^{2}$ Dustin P. Green, ${ }^{3}$ Paul B. Chen, ${ }^{2}$ Elaine D. Por, ${ }^{1}$ Nathaniel A. Jeske, $, 1,3,4$ \\ Xiaoli Gao, ${ }^{5}$ Eric R. Flores, ${ }^{1}$ and ${ }^{\circledR}$ Kenneth M. Hargreaves ${ }^{1,2,3}$ \\ ${ }^{1}$ Department of Pharmacology, ${ }^{2}$ Department of Endodontics, ${ }^{3}$ Department of Physiology, ${ }^{4}$ Department of Oral and Maxillofacial Surgery, and ${ }^{5}$ Department \\ of Biochemistry, University of Texas Health Science Center at San Antonio, San Antonio, Texas 78229
}

\begin{abstract}
Nerve growth factor (NGF) is elevated in certain chronic pain conditions and is a sufficient stimulus to cause lasting pain in humans, but the actual mechanisms underlying the persistent effects of NGF remain incompletely understood. We developed a rat model of NGFinduced persistent thermal hyperalgesia and mechanical allodynia to determine the role of transient receptor potential vanilloid 1 (TRPV1) and oxidative mechanisms in the persistent effects of NGF. Persistent thermal hypersensitivity and mechanical allodynia require de novo protein translation and are mediated by TRPV1 and oxidative mechanisms. By comparing effects after systemic (subcutaneous), spinal (intrathecal) or hindpaw (intraplantar) injections of test compounds, we determined that TRPV1 and oxidation mediate persistent thermal hypersensitivity via peripheral and spinal sites of action and mechanical allodynia via only a spinal site of action. Therefore, NGF-evoked thermal and mechanical allodynia are mediated by spatially distinct mechanisms. NGF treatment evoked sustained increases in peripheral and central TRPV1 activity, as demonstrated by increased capsaicin-evoked nocifensive responses, increased calcitonin gene-related peptide release from hindpaw skin biopsies, and increased capsaicin-evoked inward current and membrane expression of TRPV1 protein in dorsal root ganglia neurons. Finally, we showed that NGF treatment increased concentrations of linoleic and arachidonic-acid-derived oxidized TRPV1 agonists in spinal cord and skin biopsies. Furthermore, increases in oxidized TRPV1-active lipids were reduced by peripheral and spinal injections of compounds that completely blocked persistent nociception. Collectively, these data indicate that NGF evokes a persistent nociceptive state mediated by increased TRPV1 activity and oxidative mechanisms, including increased production of oxidized lipid TRPV1 agonists.
\end{abstract}

Key words: allodynia; arachidonic acid; hyperalgesia; linoleic acid; nerve growth factor; TRPV1

\section{Introduction}

Pain management is a major problem, in part due to incomplete understanding of pain mechanisms. Many studies have investigated the mechanisms for acute activation of nociceptors, but there is a large gap in knowledge regarding the mechanisms underlying the transition to chronic pain. In adults, neurotrophic factors such as nerve growth factor (NGF) regulate gene expression after nerve injury or prolonged inflammation, which can lead to the development of persistent pain (Zhu and Oxford, 2011). Endogenous NGF levels are elevated in certain chronic

\footnotetext{
Received Sept. 26, 2014; revised April 30, 2015; accepted May 1, 2015.

Author contributions: M.A.E., S.R., D.P.G., N.A.J., X.G., and K.M.H. designed research;M.A.E., P.B.C., E.D.P., N.A.J., X.G., and E.R.F. performed research; M.A.E., S.R., D.P.G., E.D.P., N.A.J., X.G., E.R.F., and K.M.H. analyzed data; M.A.E. and K.M.H. wrote the paper.

This work was supported by the National Institutes of Health (NIH Grants R01NS072890, R01GM106075, F31NS083358, and R01NS082746), the Baker Foundation, and Owen's Foundation. E.R.F. was supported by the South Texas Advanced Research Training Undergraduate Program (START-UP) funded by the NIH R25 Blueprint Program for Enhancing Neuroscience Diversity through Undergraduate Research Education Experiences (BP ENDURE Grant R25 NS080684). We thank Armen Akopian and Mayur Patil for advice and assistance. Mass spectrometry analyses were conducted in the Metabolomics Core Facility of the Mass Spectrometry Laboratory at the University of Texas Health Science Center at San Antonio, with instrumentation funded in part by NIH Grant 1S10RR031586-01. The authors declare no competing financial interests.

Correspondence should be addressed to Kenneth Hargreaves, University of Texas Health Science Center at San Antonio, 7703 Floyd Curl Drive, San Antonio, Texas 78229-3900. E-mail: Hargreaves@UTHSCSA.edu.

DOI:10.1523/JNEUROSCI.3993-14.2015

Copyright $\odot 2015$ the authors $\quad 0270-6474 / 15 / 358593-11 \$ 15.00 / 0$
}

pain conditions (Aloe et al., 1992; Iannone et al., 2002; Sarchielli et al., 2007) and a single administration of NGF is sufficient to trigger transition to a persistent pain state in humans that lasts for weeks (Petty et al., 1994; Dyck et al., 1997; Svensson et al., 2003; Rukwied et al., 2010). NGF is also synthesized and released upon single or repeated tissue injury/inflammation (Paterson et al., 2009). Moreover, tyrosine receptor kinase A (TrkA) receptor activation by NGF in immune cells evokes a feedforward release of additional NGF, together with other inflammatory mediators (Woolf et al., 1996; Nicol and Vasko, 2007; Mantyh et al., 2011). Therefore, NGF has a complex role in the development of chronic pain conditions. Compounds that inhibit NGF actions show efficacy in treating certain clinical pain disorders (Cattaneo, 2010). However, there is a critical gap in knowledge concerning mechanisms by which NGF triggers the transition to a persistent pain state.

Recent studies have implicated the transient receptor potential vanilloid 1 (TRPV1) ion channel in mediating NGF-induced persistent mechanical and thermal hypersensitivity in rats (Mills et al., 2013). NGF acutely modulates TRPV1 activity (Zhu et al., 2004; Zhu and Oxford, 2007), but the mechanism(s) mediating persistent effects are not fully understood. Studies from many laboratories, including our own, demonstrate that oxidized lipids from linoleic acid (LA) (Patwardhan et al., 2009; Patwardhan et 
al., 2010; Ruparel et al., 2012a) or arachidonic acid (AA) (Hwang et al., 2000; Gregus et al., 2012; Wen et al., 2012) activate TRPV1, resulting in nociceptor excitation and pain. LA and AA are oxidized by certain cytochrome p450s (CYPs) lipoxygenases (LOXs), cyclooxygenases (COXs), and free radicals into active metabolites (Bylund et al., 1998; Gregus et al., 2012; Ruparel et al., 2012a; Ruparel et al., 2012b; Wen et al., 2012). Injections of these oxidized linoleic acid metabolites (OLAMs) into rodent hindpaws elicit spontaneous nocifensive behavior and thermal hyperalgesia via a TRPV1-dependent mechanism (Patwardhan et al., 2010). In this study, we tested the hypothesis that NGF triggers the development of a persistent thermal and mechanical nociceptive state mediated by oxidative processes leading to increased production of endogenous TRPV1 agonists and activation of the channel.

\section{Materials and Methods}

Animals. Adult male Sprague Dawley rats (Charles River) were used for all studies. All experimental protocols were approved by the Institutional Animal Care and Use Committee of the University of Texas Health Science Center at San Antonio and conformed to the International Association for the Study of Pain and federal guidelines.

Materials. NGF-7S from murine submaxillary gland, anisomycin, cycloheximide, $\mathrm{N}$-acetylcysteine (NAC), and capsaicin were purchased from Sigma-Aldrich. Nordihydroguaiaretic acid (NDGA) and capsazepine (CPZ) were purchased from Tocris Bioscience. Bromoenol lactone (BEL) was purchased from Cayman Chemical. NGF was dissolved in PBS containing $2 \%$ bovine serum albumin. Anisomycin was dissolved in $2 \%$ $10 \mathrm{~N} \mathrm{HCl} / \mathrm{PBS}$, cycloheximide was dissolved in double distilled water, and NAC was dissolved in PBS. For behavioral experiments, capsaicin stock was made in $5 \%$ dimethylsulfoxide (DMSO)/5\% Tween/PBS that was further diluted in PBS. For electrophysiology experiments, capsaicin was dissolved in ethanol to a stock concentration of $100 \mathrm{~mm}$ that was further diluted in standard extracellular solution on the day of the experiment. NDGA was dissolved in 32\% methylpyrrolidinone/PBS. CPZ was dissolved in 20\% DMSO/80\% mineral oil.

Rat model of persistent nociception. Rats received daily systemic subcutaneous injections of NGF ( $30 \mu \mathrm{g} / \mathrm{kg} / \mathrm{d})$ or vehicle into the dorsal skin of the neck for 5 consecutive days. Interventional experiments were performed on day 8 , which was $3 \mathrm{~d}$ after the last NGF injection.

Administration of drugs. Test compounds were administered via subcutaneous injection into the dorsal neck skin ( $250 \mu \mathrm{l}$ volume), intraplantar injection into the hindpaw ( $50 \mu$ l volume), or by intrathecal injection into the lumbar region of the spinal cord (10 $\mu$ l volume). Animals were lightly restrained before subcutaneous and intraplantar injections and lightly anesthetized with isoflurane for freehand intrathecal injections, as described previously (Fairbanks, 2003; Patwardhan et al., 2009). Animals that failed to show a tail flick upon intrathecal injection or that failed to recover within $2 \mathrm{~min}$ of anesthesia were excluded from the experiment. Anisomycin $(50 \mathrm{mg} / \mathrm{kg}$, s.c.) or vehicle was administered concurrently with NGF and $6 \mathrm{~h}$ after NGF injection on each day during days 1-5. Cycloheximide ( $1 \mathrm{mg} / \mathrm{kg}$, s.c.) or vehicle was administered concurrently with NGF on each day during days $1-5$. NDGA (40 mg/kg, s.c.) or vehicle was administered $1 \mathrm{~h}$ before testing on day 8 . NAC $(50 \mathrm{mg} / \mathrm{kg}$, s.c. $)$ or vehicle was administered $30 \mathrm{~min}$ before testing on day 8 . CPZ (100 mg/ $\mathrm{kg}$, s.c.) or vehicle was administered $2 \mathrm{~h}$ before testing on day 8 . Intraplantar injection of BEL, CPZ, NDGA, or vehicle was administered 30 min before testing and intrathecal injections were administered $15 \mathrm{~min}$ before behavioral testing. Pilot studies were conducted to ensure that all compounds were tested at the time of maximal effect on behavior after subcutaneous, intraplantar, or intrathecal injection.

Behavioral assays. Blinded observers conducted all behavioral experiments. Hindpaw thermal and mechanical thresholds were assessed using the radiant heat test (Hargreaves et al., 1988) and the Ugo Basile dynamic plantar aesthesiometer (Gibbs et al., 2006), respectively. Animals were allowed to acclimate to the testing room and the testing apparatus for at least $30 \mathrm{~min}$ before the experiment. All animals tested with the radiant heat test were also tested with the dynamic plantar aesthesiometer to minimize animal use.
Spontaneous nocifensive behaviors were measured as described previously (Ruparel et al., 2008) to study the effect of capsaicin injected into the hindpaw $(1 \mu \mathrm{g})$ or intrathecal space $(0.2 \mu \mathrm{g})$. Nocifensive behavior after peripheral injection was defined as spontaneous lifting, flinching, or licking of the hindpaw and was collected in 2 min bins for $12 \mathrm{~min}$ after capsaicin or vehicle injection. Nocifensive behavior from compounds delivered into the spinal cord were defined as contracture of the caudal portions of the body, scratching and biting of the caudal portions of the body, and lifting or flinching the hind limbs, as described previously (Yaksh et al., 1979), and were collected in $2 \mathrm{~min}$ bins for $20 \mathrm{~min}$ after recovery from anesthesia.

ELISA. Plasma concentrations of NGF were measured from untreated and NGF-treated rats on days 5 and 8 . Animals were killed by decapitation and trunk blood was collected into heparinized collection tubes. Blood was centrifuged at $200 \times g$ for 15 min to isolate plasma, followed by centrifugation at $2000 \times g$ for $15 \mathrm{~min}$ to isolate platelet poor plasma (PPP) for use with the ChemiKine NGF Sandwich ELISA kit (Millipore) (Blandini et al., 2006). PPP was used immediately or stored at $-80^{\circ} \mathrm{C}$ until use. Linear regression was used to generate a standard curve $\left(r^{2}\right.$ $\geq 0.99)$.

Primary sensory neuron culture. Animals were killed by decapitation and lumbar (L4-L6) dorsal root ganglia (DRG) were removed within 15 min and placed into ice-cold HBSS (Invitrogen). DRG neurons were separated in $1 \mathrm{mg} / \mathrm{ml}$ collagenase-dispase solution and plated onto poly D-lysine-laminin-coated coverslips (Clontech). Cells were maintained in minimal media containing DMEM, supplemented with $2 \%$ fetal bovine serum, $2 \mathrm{~mm}$ L-glutamine, $100 \mathrm{U} / \mathrm{ml}$ penicillin, and $100 \mu \mathrm{g} / \mathrm{ml}$ streptomycin. NGF was not added to the medium to avoid any confounding effects of previous daily, systemic NGF treatment. Experiments were conducted within $4-6 \mathrm{~h}$ of plating.

Electrophysiology. Recordings were made in whole-cell patch-clamp (holding potential of $-60 \mathrm{mV}$ ) configuration at $22-24^{\circ} \mathrm{C}$ from the somata of small- to medium-sized neurons $(15-40 \mathrm{pF})$. Data were acquired and analyzed using an Axopatch 200B amplifier and pCLAMP10.0 software (Molecular Devices). Recording data were filtered at $0.5-2.5 \mathrm{kHz}$ and sampled at $2-10 \mathrm{kHz}$. Borosilicate pipettes with filaments (Sutter Instruments) were polished to resistances of 3-4 $\mathrm{M} \Omega$ in whole-cell pipette solution. Access resistance $\left(R_{\mathrm{s}}\right)$ was compensated $40-80 \%$ when appropriate up to the value of 7-10 M $\Omega$. Data were rejected when $R_{\mathrm{s}}$ changed $>20 \%$ during recording, leak currents were $>50 \mathrm{pA}$, or input resistance was $<300 \mathrm{M} \Omega$. Standard external solution contained the following (in mM): $140 \mathrm{NaCl}, 5 \mathrm{KCl}, 2 \mathrm{CaCl}_{2}, 1 \mathrm{MgCl}_{2}, 10 \mathrm{D}$-glucose, and 10 HEPES, $\mathrm{pH}$ 7.4. Standard pipette solution contained the following (in mM): $140 \mathrm{KCl}, 1 \mathrm{MgCl}_{2}, 1 \mathrm{CaCl}_{2}, 10$ EGTA, 10 D-glucose, and $10 \mathrm{HEPES}$, $\mathrm{pH}$ 7.3. Capsaicin was applied using a fast, pressure-driven and computer-controlled 5-channel system (MicroData Instrument).

Skin superfusion and radioimmunoassay. Six millimeter biopsies of plantar hindpaw skin (in the same area stimulated in the radiant heat test) were collected and placed into 24-well plates (two biopsies/well) containing HBSS for $30 \mathrm{~min}$ for equilibration. After baseline sample collection, capsaicin was applied for $2 \mathrm{~min}$ with collection of superfusate for $20 \mathrm{~min}$ and CGRP measurement was conducted by radioimmunoassay as described previously (Ruparel et al., 2008).

Crude plasma membrane preparation. DRG were collected and homogenized by 20 strokes in a Potter-Elvehjem homogenizer in a hypotonic buffer containing the following (in mM): 25 HEPES, 25 sucrose, 1.5 $\mathrm{MgCl}_{2}$, and $50 \mathrm{NaCl}, \mathrm{pH}$ 7.2. The cell extract was incubated on ice for 15 $\mathrm{min}$ and then centrifuged at $1000 \times \mathrm{g}$ for $1 \mathrm{~min}$ at $4^{\circ} \mathrm{C}$ to remove nuclei and unlysed cells from the homogenate. The resulting supernatant was centrifuged at $16,000 \times g$ for $30 \mathrm{~min}$ at $4^{\circ} \mathrm{C}$, separating cytosolic proteins from cell membrane proteins. The pellet (crude membrane fraction) was then resuspended in $400 \mu \mathrm{l}$ of homogenization buffer containing $1 \%$ Triton X-100.

Western blot. Protein quantification of plasma membrane and cytosolic homogenates was performed using the Bradford assay (Bradford, 1976) (Sigma-Aldrich) following the manufacturer's directions. Samples $(50 \mu \mathrm{g})$ were resolved via $15 \%$ SDS-PAGE, and transferred to PVDF membranes (Millipore). Western blots were blocked in 5\% nonfat milk in Tris-buffered saline/Tween-20 and visualized using anti-TRPV1, anti- 
$\beta 1$-integrin or anti- $\beta$-actin primary antibodies (Santa Cruz Biotechnology), followed by the appropriate horseradish peroxidase-conjugated secondary antisera (GE Healthcare) and enhanced chemiluminescence detection following the manufacturer's instructions (GE Healthcare). Densitometry measurements were determined using ImageJ version 1.62 , with the reported pixel density $=$ band density - lane background density. Densitometry measurements for immunoreactive TRPV1 from crude PM fractions were normalized to those for $\beta 1$-integrin and TRPV1 from cytosolic fractions were normalized to $\beta$-actin.

HPLC electrospray ionization tandem MS. For HPLC electrospray ionization tandem MS, polyunsaturated fatty acids (PUFAs) were extracted from weighed samples (containing at least $25 \mathrm{mg}$ ) of spinal cord and $6 \mathrm{~mm}$ hindpaw skin biopsies using 100\% ice-cold methanol. Stable isotope-labeled standards (arachidonic acid-d8, 12S-hydroxyeicosatetraenoic acid-d8 (12SHETE-d8), and 9S-hydroxyoctadecadienoic acid-d4 (9S-HODE-d4)) were added at the time of extraction for absolute quantification. Tissue samples with the solvents were homogenized with an Omni Bead Ruptor Homogenizer (OMNI International). MS analyses were conducted on a Thermo Fisher Q Exactive fitted with a PicoChip nanospray source (New Objective) and a PicoChip column (Waters Atlantis dC18 column; 150 $\mu \mathrm{m} \times 105 \mathrm{~mm} ; 3 \mu \mathrm{m}$ particles). A $55 \mathrm{~min}$ water/acetonitrile/isopropanol/ammonium acetate gradient was run at the flow rate of $1 \mu \mathrm{l} / \mathrm{min}$. Mobile phase A is acetonitrile/water (40:60) containing $10 \mathrm{~mm}$ ammonium acetate and mobile phase $B$ is acetonitrile/isopropanol (10:90) containing $10 \mathrm{~mm}$ ammonium acetate. Data-dependent analyses were conducted using one full MS scan (70,000 resolution) followed by six tandem MS scans with electrospray negative ion detection. Standard curves were generated for all targeted PUFAs using appropriate stable isotope labeled internal standards and authentic fatty acids. Quantitative results were obtained by reference of the experimental peak area ratios to the standard curves.

Data analysis. Nonlinear regression and GLM methods were used for statistical analysis, including ANOVA and Student's $t$ test, with Tukey's post hoc analyses in studies powered to generate $80 \%$ power for detecting a $25 \%$ difference between groups at $p<0.05$, with variance estimates derived from previous studies in our laboratory. Error bars in figures are $\operatorname{SEM}\left({ }^{\star} p<0.05,{ }^{* *} p<0.01,{ }^{* * *} p<0.001\right)$.

\section{Results \\ NGF-induced persistent thermal and mechanical nociception requires protein synthesis and are mediated by TRPV1 and oxidation}

A model of NGF-induced persistent nociception was developed by administering systemic NGF ( $30 \mu \mathrm{g} / \mathrm{kg} / \mathrm{d}$, s.c.) for $5 \mathrm{~d}$ to male rats (Fig. 1). By day 5, NGF-treated animals exhibited significant thermal hyperalgesia compared with vehicle-treated rats and hyperalgesia was maintained through days 8-11 (Fig. 1A). Similarly, NGF treatment caused significant mechanical allodynia by day 5 that was maintained through days 8-11 (Fig. 1B). Consistent with the clinical observation that NGF is a sufficient stimulus to cause lasting pain in humans (Petty et al., 1994; Svensson et al., 2003; Rukwied et al., 2010), these data demonstrate that systemic NGF causes thermal and mechanical nociception in rats that persists days after treatment cessation.

The persistent nociception observed at day 8 is presumably due to the downstream effects of receptor activation during NGF treatment. Because NGF has a circulating half-life of $4.5 \mathrm{~h}$ in rats (Tria et al., 1994), studies conducted on day 8 were $\sim 16$ half-lives after NGF administration (i.e., we estimated that $\sim 99.998 \%$ of circulating NGF was eliminated). To exclude directly the possibility of residual exogenous NGF, we measured circulating levels of NGF ( $n=8 /$ group) by ELISA. There were no detectable (i.e., $<10 \mathrm{pg} / \mathrm{ml}$ ) levels of NGF in plasma from untreated and NGFtreated animals on day 8. In contrast, NGF-treated animals on day 5 had plasma NGF concentrations of $41.2 \pm 9.0 \mathrm{pg} / \mathrm{ml}$. These data indicate that day 8 observations represent continued effects of NGF that persist long after clearance of the neurotrophin. We next determined whether changes in protein expression were required for persistent effects of NGF. We coadministered NGF together with either anisomycin or cycloheximide $(n=6-10 /$ group) during days $1-5$ and measured nociception on day 8 . Anisomycin and cycloheximide cotreatment significantly blocked the development of both thermal hyperalgesia (Fig. 1C) and mechanical allodynia (Fig. 1D), demonstrating that de novo protein synthesis is required for the persistent effects of NGF. Together, these findings demonstrate that day 8 nociception in the NGF model represent persistent pain behaviors that require changes in protein expression and persist beyond physical elimination of the exogenous neurotrophin.

Prior studies have shown that NGF modulates TRPV1 acutely (Zhu et al., 2004; Zhu and Oxford, 2007); therefore, we investigated whether NGF exerts persistent effects on TRPV1. First, we evaluated whether inhibition of TRPV1 could reduce persistent nociception from NGF treatment. Systemic injection of the TRPV1 antagonist capsazepine (CPZ, $100 \mathrm{mg} / \mathrm{kg}$, s.c.) significantly and rapidly inhibited thermal and mechanical nociception (Fig. 1C,D), providing a direct link between TRPV1 channel activation and persistent pain behaviors. Because certain oxidized lipids comprise a population of endogenous TRPV1 activators, we next evaluated whether oxidative mechanisms contribute to nociceptive behaviors caused by NGF. Administration of the combined oxidative enzyme inhibitor/antioxidant NDGA (40 $\mathrm{mg} / \mathrm{kg}$, s.c.) significantly reduced thermal and mechanical nociception (Fig. $1 C, D$ ), implicating a definitive role for oxidative processes in persistent nociception from NGF. NDGA is a broadbased CYP and LOX inhibitor as well as a potent antioxidant (Goodman et al., 1994; DuPont et al., 2001) that reduces formation of TRPV1-active oxidized lipids (Patwardhan et al., 2010), whereas NAC is a thiol-containing antioxidant that reacts directly with free radicals and has been shown to modulate TRPV1 activity (Nazıroğlu et al., 2013). In contrast to NDGA, systemic NAC injection failed to reduce thermal hypersensitivity or mechanical allodynia caused by NGF (Fig. 1C,D), suggesting that enzymatic oxidation contributes preferentially to persistent pain behaviors over nonenzymatic free radical oxidation. Collectively, these systemic administration studies indicate that persistent NGF nociception requires de novo protein synthesis and is mediated by TRPV 1 and enzymatic oxidative processes. However, systemic injection of compounds provides little insight into sites of action where NGF mediates persistent nociception. Accordingly, the next series of experiments used localized routes of injection to isolate and study peripheral and central mechanisms that contribute to NGF-evoked persistent nociception.

\section{NGF-evoked thermal hyperalgesia and mechanical allodynia are mediated by spatially distinct mechanisms}

Previous studies have suggested that both peripheral and central mechanisms contribute to NGF-induced pain behaviors (Lewin et al., 1993; Lewin et al., 1994). Accordingly, we compared responses to compounds injected into either intraplantar hindpaw or intrathecal spinal tissues. Peripheral (intraplantar) administration of CPZ and NDGA significantly and dose-dependently reversed NGF-evoked thermal hyperalgesia (Fig. 2A). Control experiments verified that these effects were peripherally mediated because intraplantar injection of these drugs into one hindpaw had no effect on thresholds in the contralateral hindpaw (data not shown). Moreover, spinal injection of CPZ and NDGA also significantly reversed NGF-evoked thermal hyperalgesia (Fig. 2C). Together, these data indicate that blockade of TRPV1 or oxida- 
A

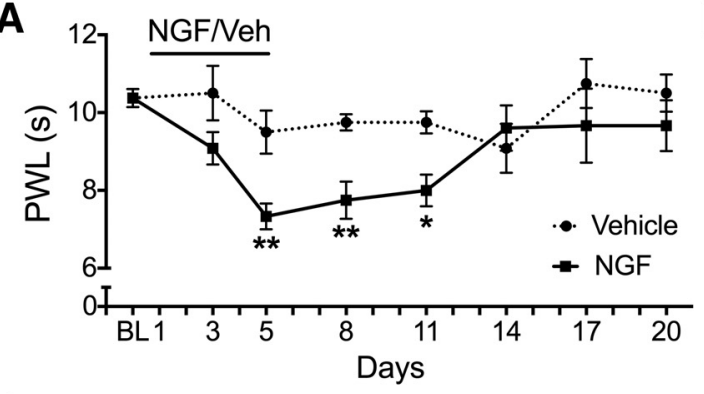

B

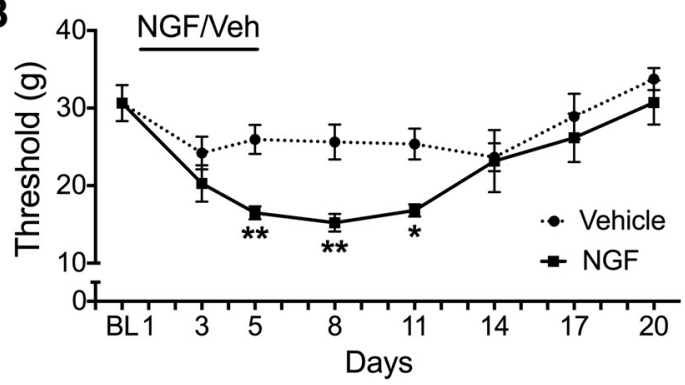

C 157
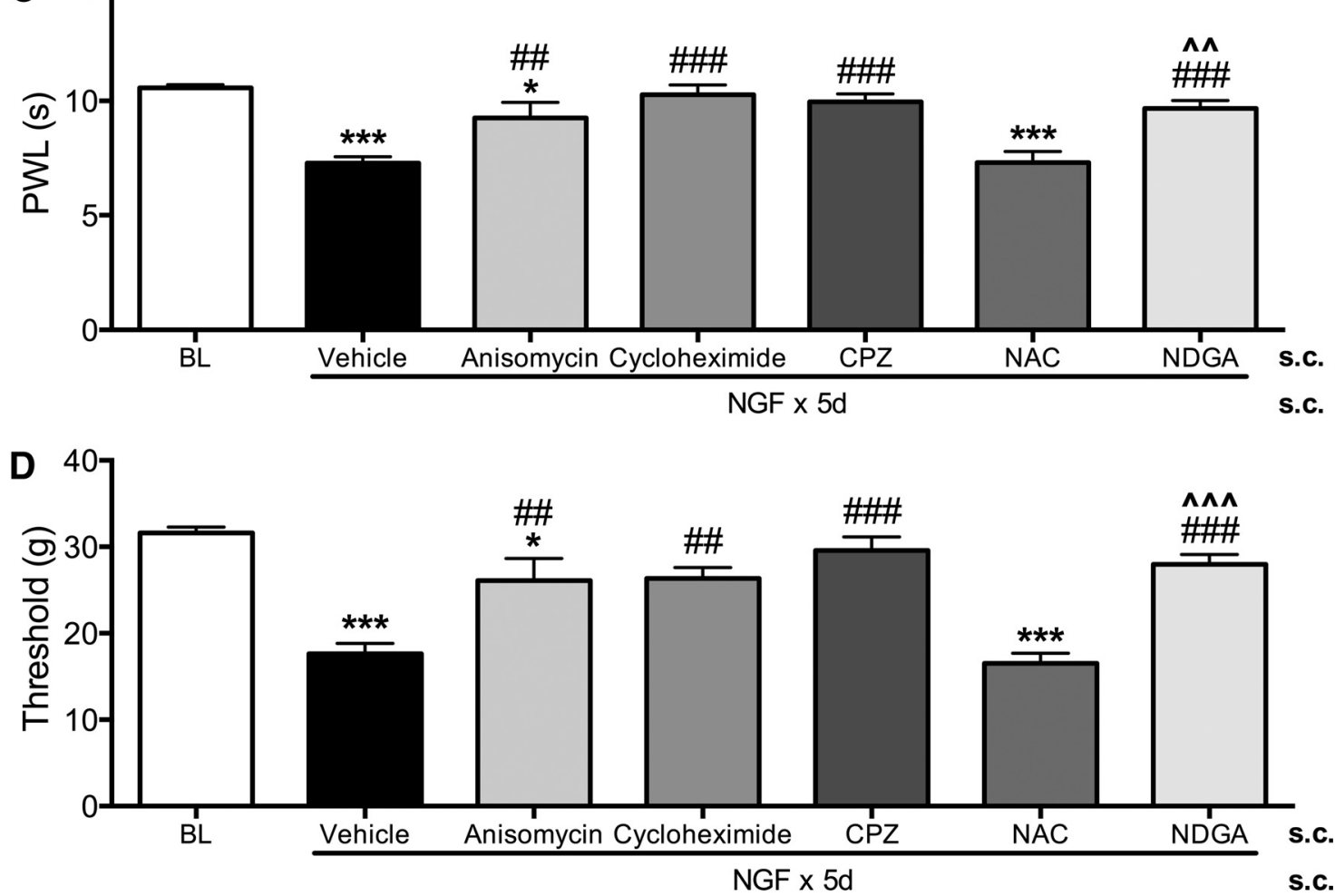

Figure 1. NGF-induced persistent thermal and mechanical allodynia require protein synthesis and are mediated by TRPV1 and oxidation. Blinded observers measured thermal $(\boldsymbol{A})$ and mechanical hindpaw thresholds $(\boldsymbol{B})$ for 2 weeks in NGF- $(30 \mu \mathrm{g} / \mathrm{kg} / \mathrm{d} \times 5 \mathrm{~d}$ ) or vehicle ( $n=6 / \mathrm{group})$-treated rats. Data were analyzed by repeated-measures ANOVA with Tukey's post hoc test. Error bars are SEM. ${ }^{*} p<0.05,{ }^{* *} p<0.01,{ }^{* * *} p<0.001$. Blinded observers measured thermal $(\boldsymbol{C})$ and mechanical (D) thresholds in NGF-treated rats on day 8 after coadministration of anisomycin ( $50 \mathrm{mg} / \mathrm{kg}$, s.c.), cycloheximide (1 mg/kg, s.c.), or vehicle with NGF during days $1-5$ or after CPZ (100 mg/kg, s.c.), NDGA (40 mg/kg, s.c.), NAC ( $50 \mathrm{mg} / \mathrm{kg}$, s.c.), or vehicle injection on day 8 ( $n=6-10 / \mathrm{group})$. Data were analyzed by ANOVA with Tukey's post hoc test. Error bars are SEM. ${ }^{*} p<0.05,{ }^{* * *} p<0.001$ compared with baseline; \#\#p $<0.01$, \#\#p $<0.001$ compared with vehicle; $\wedge \wedge p<0.01$, $\wedge \wedge \wedge p<0.001$ compared with NAC.

tive enzymes, in either spinal cord or hindpaw skin, is sufficient to reduce NGF-induced thermal hyperalgesia. However, a different pattern was observed with NGF-mediated mechanical allodynia because central administration of either CPZ or NDGA significantly reversed mechanical allodynia, whereas peripheral injection had no effect (Fig. $2 B, D$ ). Notably, the peripheral doses of CPZ and NDGA were biologically active because they significantly reversed thermal hyperalgesia (Fig. 2A). Therefore, NGF-evoked thermal and mechanical nociception are mediated by spatially distinct mechanisms: thermal nociception is mediated in both peripheral tissues and spinal cord, whereas mechanical nociception is mediated exclusively by spinal mechanisms.

Overall, these findings demonstrate that TRPV1 and oxidation are key mechanisms and convergence points for both persistent thermal and mechanical nociception after NGF despite the fact that spatially distinct mechanisms mediate each type of nociception. Furthermore, the full efficacy and similarities in results from peripheral versus spinal routes of administration between $\mathrm{CPZ}$ and NDGA are consistent with the hypothesis that NGF causes persistent nociception through increased TRPV1 activation by oxidized lipid agonists. Although enhanced enzymatic production of oxidized lipid LA and AA metabolites is a possible mechanism to connect TRPV1 and oxidative mechanisms functionally, behavioral studies using CPZ and NDGA are insufficient to test this hypothesis adequately. Phospholipase A2 (PLA2) enzymes release fatty acids from membrane phospholipids (Konkel and Schunck, 2011), so we used the PLA2 inhibitor BEL to evaluate the effect of blocking free fatty acid release on NGF-induced persistent nociception. Peripheral (intraplantar) administration of BEL significantly and dose-dependently reversed NGF-evoked thermal hyperalgesia (Fig. 2E), with no effect on mechanical allodynia (Fig. $2 F$ ), whereas spinal (intrathecal) injection of BEL significantly and dose-dependently reversed both thermal hyperalgesia and mechanical allodynia (Fig. 2G,H). The similarity in efficacy of BEL, CPZ, and NDGA is consistent with the hypothe- 
A

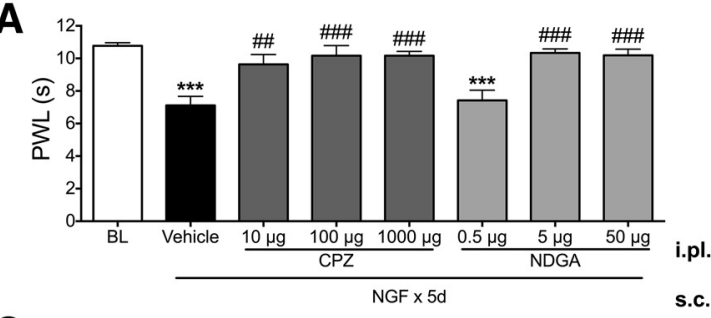

C

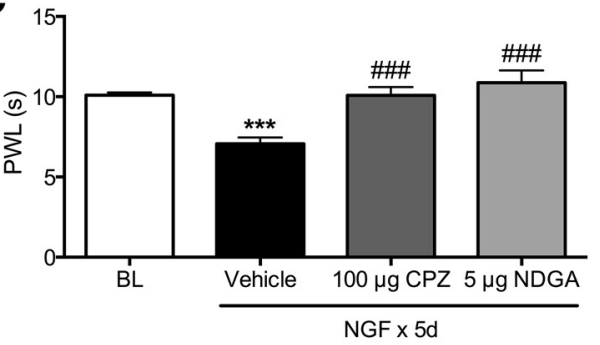

E

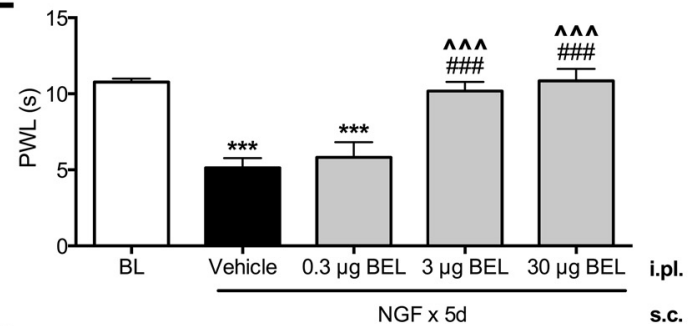

G

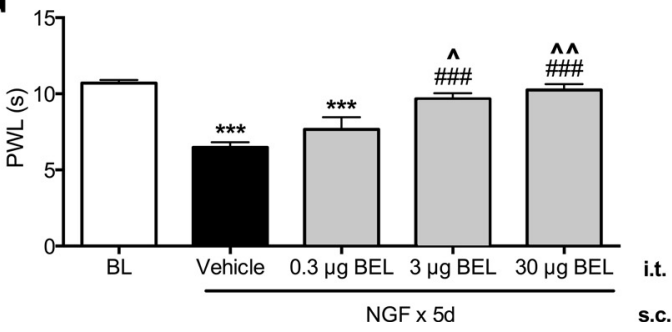

B

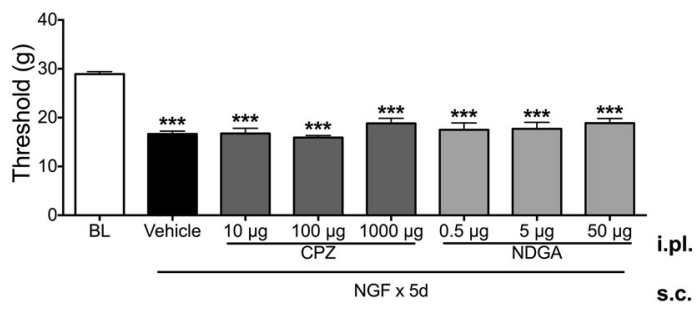

D

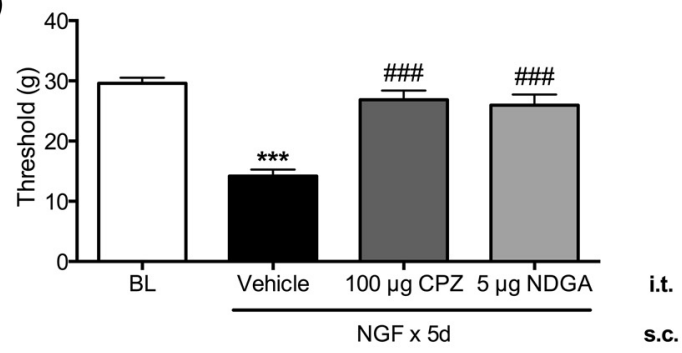

F

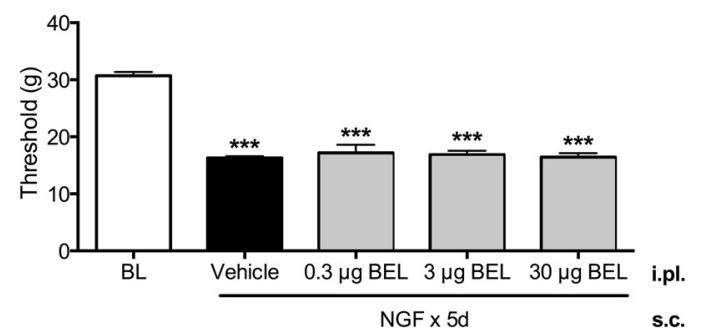

H

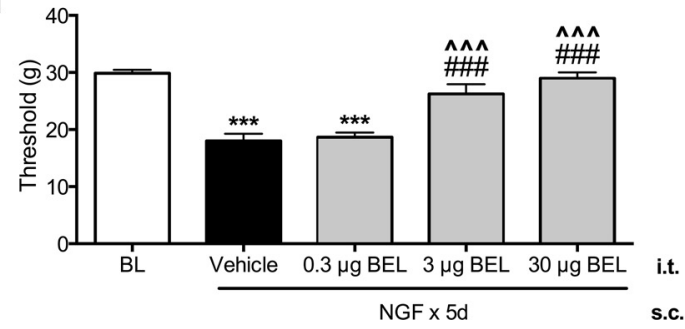

Figure 2. Persistent NGF-evoked thermal hyperalgesia and mechanical allodynia are mediated by spatially distinct mechanisms. Blinded observers measured thermal $(\boldsymbol{A})$ and mechanical $(\boldsymbol{B})$ thresholds in NGF-treated rats on day 8 after hindpaw injections of NDGA $(0.5,5$, or $50 \mu \mathrm{g}$, i.pl.), CPZ (10, 100, or $1000 \mu \mathrm{g}$, i.pl.), or vehicle $(n=5-6 /$ group). Blinded observers measured thermal (C) and mechanical (D) thresholds in NGF-treated rats on day 8 after spinal cord injections of NDGA (5 $\mu$ g, i.t.), CPZ (100 $\mu \mathrm{g}$, i.t.), or vehicle ( $n=5-6 /$ group). Data were analyzed by ANOVA with Tukey's post hoc test. Error bars are SEM. ${ }^{* * *} p<0.001$ compared with baseline, \#\#p $<0.01$, \#\#\#p $<0.001$ compared with vehicle. Blinded observers measured thermal $(\boldsymbol{E})$ and mechanical $(\boldsymbol{F})$ thresholds in NGF-treated rats on day 8 after hindpaw injections of BEL $(0.3,3$, or $30 \mu \mathrm{g}$, i.pl.) or vehicle ( $n=6-7 /$ group). Blinded observers measured thermal $(\boldsymbol{G})$ and mechanical $(\boldsymbol{H})$ thresholds in NGF-treated rats on day 8 after spinal cord injections of BEL $\left(0.3,3\right.$, or $30 \mu$ g, i.t.) or vehicle $\left(n=6-7 /\right.$ group). Data were analyzed by ANOVA with Tukey's posthoc test. Error bars are SEM. ${ }^{* * *} p<$ 0.001 compared with baseline; \#\#\#p 0.001 compared with vehicle; $\wedge p<0.05, \wedge \wedge p<0.01, \wedge \wedge \wedge p<0.001$ compared with $0.3 \mu \mathrm{g}$ BEL.

sis that NGF leads to increased TRPV1 activities by release of endogenous TRP-active lipid metabolites. To test this hypothesis directly, we next evaluated persistent effects of NGF on TRPV1 activity and expression, followed by additional experiments that determined directly the effect of NGF alone and with NDGA or BEL cotreatment on levels of oxidized lipid TRPV1 agonists in spinal cord and peripheral tissues.

\section{NGF causes persistent increases in TRPV1 activities}

Because TRPV1 antagonism blocked NGF-mediated persistent nociception, we first evaluated the effects of NGF on behavioral measures of TRPV1 activity. NGF-treated animals displayed a threefold increase in nocifensive behaviors to hindpaw injection of capsaicin compared with vehicle control animals (Fig. 3A), suggesting that peripheral TRPV1 activity is persistently increased after NGF. Conversely, cotreatment of NGF with either anisomycin or cycloheximide significantly reduced hindpaw no- cifensive behaviors on day 8 (Fig. $3 A$ ), demonstrating that inhibition of protein translation during NGF treatment blocked the increases in TRPV1 activities. These findings suggest that NGF could trigger persistent nociception through changes in protein translation of peripheral TRPV1 and/or genes involved in signal transduction pathways, posttranslational modification, or surface trafficking of the receptor. Next, we determined the effect of NGF on spinal TRPV1 activity. NGF-treated rats displayed a modest, but significant, increase in nocifensive behaviors evoked by spinal (intrathecal) injection of capsaicin compared with vehicle control animals (Fig. 3B), suggesting that spinal TRPV1 activity is also enhanced after NGF. Together, these behavioral studies demonstrate that persistent NGF-induced nociception is accompanied by increases in both peripheral and central TRPV1 activity.

To investigate potential mechanisms by which TRPV1 activity was enhanced, we evaluated persistent NGF effects on capsaicin- 
A

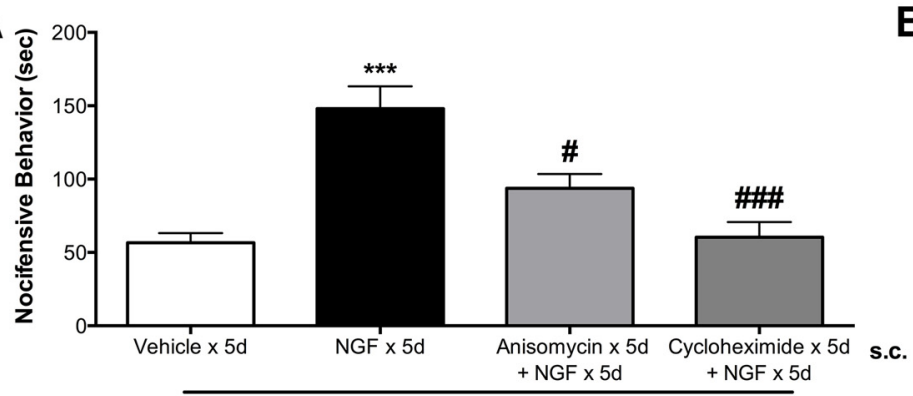

1 ug capsaicin

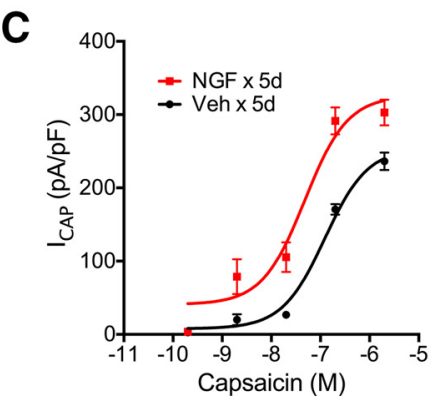

D

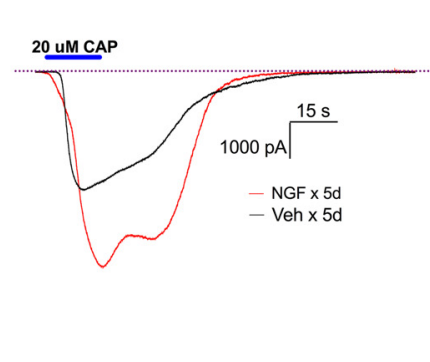

B

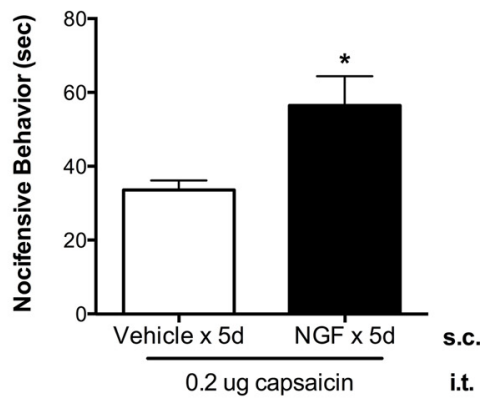

i.pl.
E

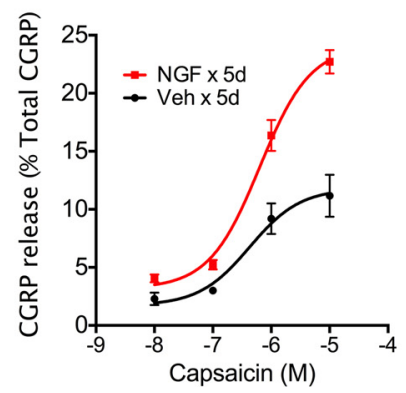

Figure 3. NGF caused persistent increases in TRPV1 activity. $A$, Blinded observers measured nocifensive behaviors evoked by peripheral capsaicin (1 $\mu$ g, i.pl.) injection on day 8 in vehicle-treated rats, NGF-treated rats, and NGF-treated rats cotreated with anisomycin $(50 \mathrm{mg} / \mathrm{kg}$, s.c. $\times 5 \mathrm{~d})$ or cycloheximide $(1 \mathrm{mg} / \mathrm{kg}, \mathrm{s.c.} \times 5 \mathrm{~d})(n=5-6 / \mathrm{group})$. Data were analyzed by ANOVA with Tukey's posthoc test. $\boldsymbol{B}$, Blinded observers measured nocifensive behaviors evoked by central injection of capsaicin $(0.2 \mu \mathrm{g}$, i.t.) on day 8 in vehicle or NGF-treated rats $(n=8-10 / \mathrm{group})$. Data were analyzed by Student's $t$ test. C, D, Capsaicin-evoked inward currents were measured from acutely cultured L4-L6 DRGs from NGF- or vehicle-treated rats on day 8 ( $n=4-6 /$ concentration/group; $C$ ) with representative traces of maximal capsaicin-evoked inward currents in DRGs from NGF- and vehicle-treated rats (D). E, Capsaicin-evoked CGRP release in $6 \mathrm{~mm}$ hindpaw skin biopsies from NGF- or vehicle-treated animals on day 8 were quantified using radioimmunoassay ( $n=6 /$ concentration/group) and CGRP release data were normalized to percentage total CGRP content to account for differences in overall CGRP content after NGF treatment. Data were analyzed by nonlinear regression. Error bars are SEM. ${ }^{*} p<0.05,{ }^{* *} p<0.01,{ }^{* * *} p<0.001$ compared with vehicle $\times 5$ d; $\# p<$ 0.05 , \#\#\#p $<0.001$ compared with NGF $\times 5 \mathrm{~d}$.

evoked inward current $\left(I_{\mathrm{CAP}}\right)$ in acutely isolated DRG neurons (Fig. 3C). NGF treatment significantly increased maximal current density $\left(E_{\max }\right)$ of $I_{\mathrm{CAP}}(\mathrm{NGF}=325.9 \pm 29.6 \mathrm{pA} / \mathrm{pF}$ vs vehicle $=254.4 \pm 13.0 \mathrm{pA} / \mathrm{pF}$; representative traces in Fig. $3 D)$ with no effect on half-maximal effect $\left(E C_{50}\right)$ concentrations (NGF $=$ $48.3 \mathrm{nM}$ vs vehicle $=123.0 \mathrm{nM}$ ). If changes in TRPV1 density in acutely cultured DRG cell bodies reflect receptor density at peripheral and central nerve terminals, then these data support the hypothesis that NGF-evoked increases in behavioral TRPV1 activity are mediated by increased TRPV1 density at peripheral and central DRG nerve terminals. As a direct approach to evaluate TRPV1 activity in peripheral terminals, we measured capsaicinevoked CGRP exocytosis in hindpaw skin biopsies taken from NGF- or vehicle-treated rats on day 8 (Fig. $3 E$ ). Similar to the electrophysiology studies, NGF treatment significantly increased maximal capsaicin-evoked CGRP release $\left(E_{\max }\right)$ values in hindpaw skin $(\mathrm{NGF}=24.1 \pm 1.1 \%$ total CGRP, vs vehicle $=11.8 \pm$ $1.3 \%$ total CGRP), with no change in $E C_{50}$ (NGF $=636.1 \mathrm{nM}$ vs vehicle $=427.4 \mathrm{~nm}$ ). The results from these electrophysiologic studies on DRG somata and exocytosis studies from cutaneous peptidergic terminals suggest that the neuronal mechanism by which NGF enhances peripheral TRPV1-mediated pain behavior is through increased TRPV1 membrane density in DRG cell bodies, peripheral nerve terminals, and possibly central nerve terminals.

NGF increases membrane TRPV1 expression in rat L4-L6 DRGs but not lumbar spinal cord

To test directly for increased TRPV1 density in DRG and spinal cord membranes, we measured membrane and cytosolic TRPV1 protein in L4-L6 DRGs and lumbar spinal cord collected from untreated or NGF-treated animals (Fig. $4 A-D$ ). Fractionated Western blot analysis indicated that NGF significantly increased membrane TRPV1 protein in DRG cell bodies on days 5 and 8, with no detectable changes in cytosolic TRPV1 protein (Fig. $4 A-$ $D)$. Surprisingly, NGF appeared to have no effect on either membrane or cytosolic TRPV1 protein in spinal cord (Fig. 4E-H), suggesting that NGF may increase TRPV1 density in DRG cell bodies and peripheral, but not central, terminals preferentially. Together, studies on TRPV1 activity and protein expression support the hypothesis that NGF enhances peripheral TRPV1mediated pain behaviors by increasing peripheral membrane TRPV1 protein density in peripheral DRG nerve terminals in hindpaw skin. Differences between the effect of NGF on TRPV1 protein in DRG cell bodies and spinal cord support the hypothesis that NGF-mediated thermal hypersensitivity and mechanical allodynia are mediated by distinct mechanisms.

\section{NGF-evoked increased concentrations of TRPV1-active oxidized lipid metabolites in lumbar spinal cord are attenuated by NDGA and BEL}

The parallel effects of BEL, CPZ, and NGDA for blocking persistent NGF nociception (Figs. 1,2) are consistent with the hypothesis that persistent NGF nociception is mediated by activation of TRPV1 by oxidized lipid agonists. Because certain oxidized metabolites of either LA (Patwardhan et al., 2009; Patwardhan et al., 2010; Ruparel et al., 2012a) or AA (Hwang et al., 2000; Gregus et al., 2012; Wen et al., 2012) activate TRPV1, we used HPLC/MS analyses to generate a lipidomic profile of NGF-evoked changes of these metabolites in L4-L6 spinal cord and determined the 
A

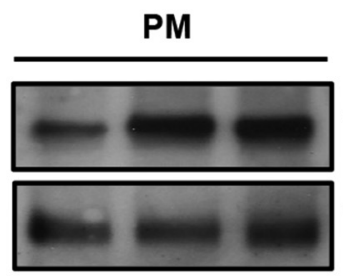

Untreated Day 5 Day 8

C

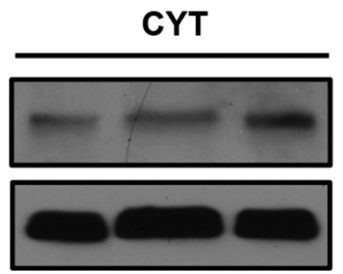

Untreated Day 5 Day 8
Untreated Day 5 Day 8

E

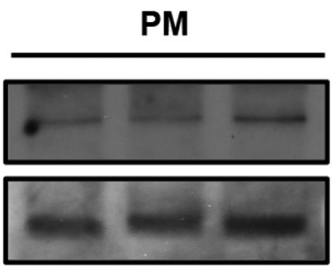

G
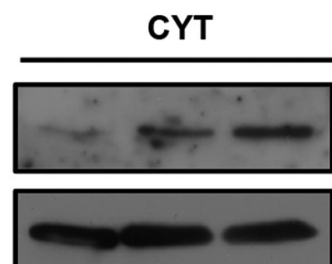

WB: $\beta$-actin

WB: TRPV1

WB: $\beta$-actin

WB: TRPV1

WB: $\beta 1$ integrin

WB: TRPV1

Untreated Day 5 Day 8
B

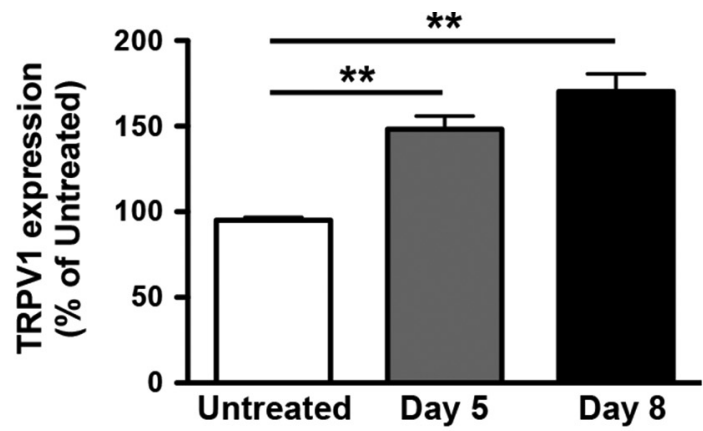

D

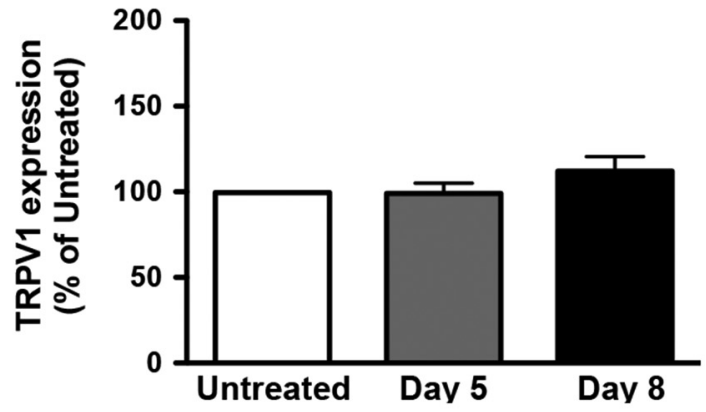

F

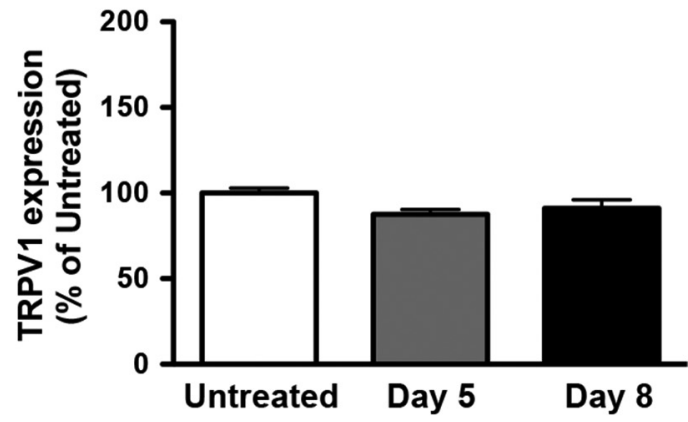

H

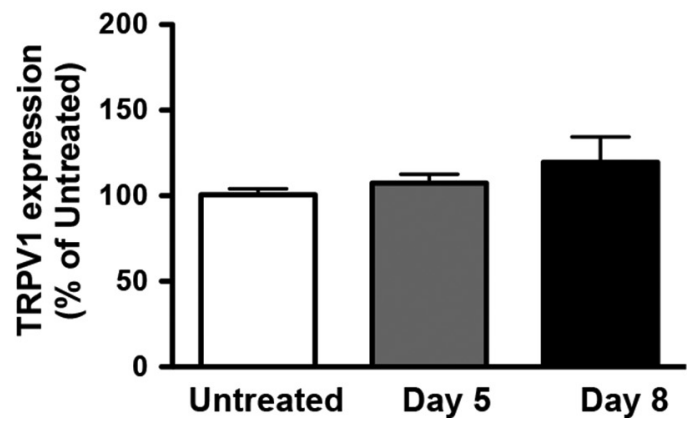

Figure 4. NGF increased membrane TRPV1 expression in rat lumbar (L4-L6) DRGs but not spinal cord. $A$, TRPV1 expression at the plasma membrane (PM) of DRGs from untreated and NGF-treated rats on days 5 and $8 . \boldsymbol{B}$, Quantification of TRPV1 expression in $\boldsymbol{A}$ compared with untreated control. C, TRPV1 expression in the cytosol (CYT) of DRG from untreated and NGF-treated rats on days 5 and 8. D, Quantification of TRPV1 expression in C compared with untreated control. $\boldsymbol{E}$, TRPV1 expression at the PM of spinal cord from untreated and NGF-treated rats on days 5 and 8 . $\boldsymbol{F}$, Quantification of TRPV1 expression in $\boldsymbol{E}$ compared with untreated control. G, TRPV1 expression in the CYT of spinal cord from untreated and NGF-treated rats on days 5 and 8 . $\boldsymbol{H}$, Quantification of TRPV1 expression in $\mathbf{G}$ compared with untreated control. Data were analyzed by ANOVA with Tukey's post hoc test. ${ }^{* *} p<0.01$. Data are representative of three independent experiments.

effects of spinal injection of NDGA and BEL on metabolite concentrations (Fig. 5). In spinal cord, NGF treatment had no effect on levels of the precursors LA or AA (Fig. $5 A$ ), but significantly increased concentrations of most of the LA metabolites measured, including 9-HODE and 13-HODE, 9-oxooctadecadienoic acid (9oxoODE) and 13-oxoODE, 9(10)-epoxyoctadecenoic acid (9(10)EpOME) and 12(13)-EpOME, and 9,10-dihydroxyoctadecenoic acid (9,10-DiHOME), as well as the AA metabolites 15-HETE, 5-HETE, and leukotriene B4 (LTB4) (Fig. 5B-D). Moreover, intrathecal injection of NDGA significantly reduced NGF-evoked increases in all LA and AA metabolites with the exception of 13-oxoODE (Fig. 5B-D). Interestingly, BEL reduced NGFevoked elevations in LA metabolites, but had no effect on NGFevoked increases in AA metabolite concentrations (Fig. $5 B-D)$, suggesting that NDGA and BEL alter metabolite formation in the spinal cord by targeting different metabolic pathways. The reductions in NGF-evoked increases in TRPV1-active oxidized lipids by NDGA and BEL is quite similar to their effects on reducing nociception (Fig. 2). Because BEL reduced linoleic acid metabolites, but not AA metabo- 
A

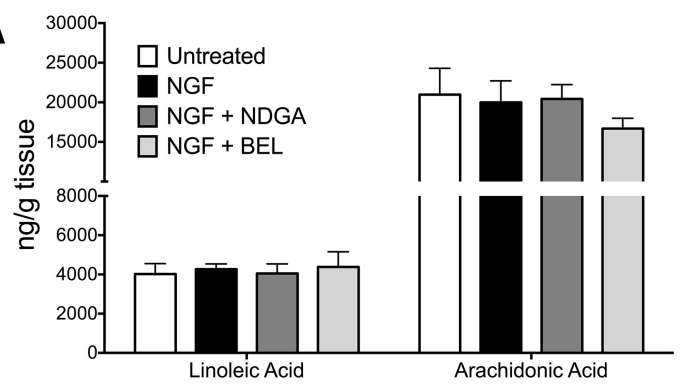

C

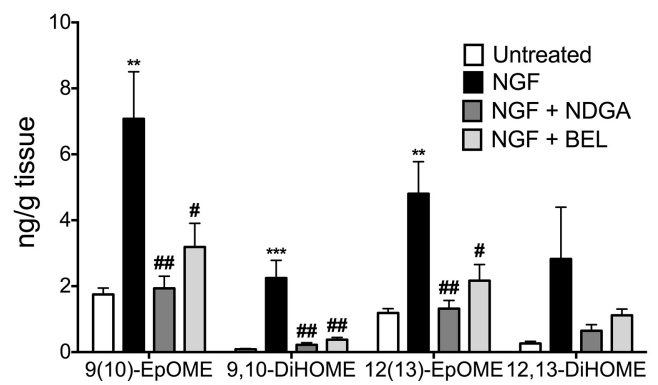

B

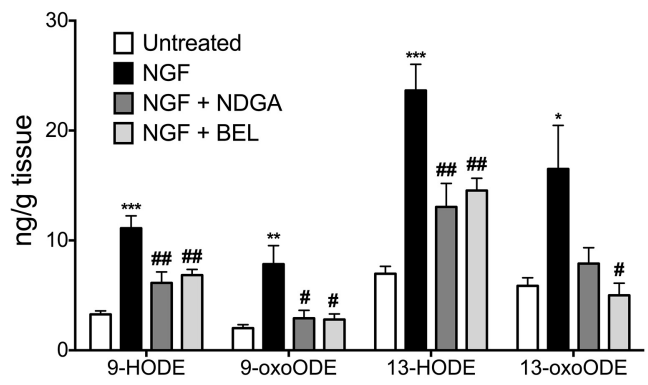

D

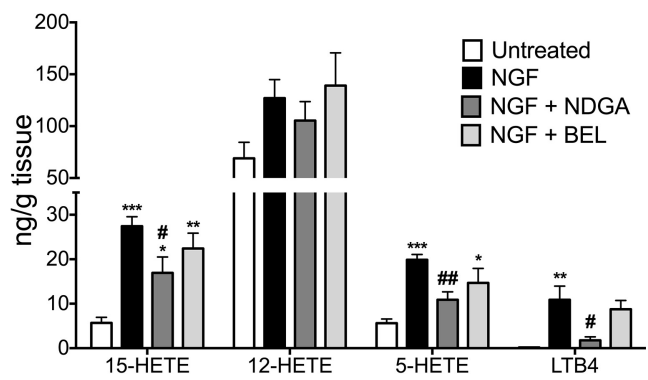

Figure 5. NGF-evoked increases in TRPV1-active oxidized lipids in spinal cord were attenuated by spinal injection of NDGA and BEL. Lumbar spinal cord were rapidly removed from untreated rats, NGF-treated rats, and NGF-treated rats cotreated with NDGA (5 $\mu$ g, i.t.) or BEL (30 $\mu$ g, i.t.) on day 8 ( $n=7-10 /$ group). Lipids were extracted in methanol-containing isotope-labeled standards for MS analysis of LA and AA ( $\boldsymbol{A})$, HODE and oxOODE metabolites (B), EpOME and DiHOME metabolites ( $)$, and HETE metabolites and LTB4 in spinal cord tissues ( $\boldsymbol{D}$ ). Quantitative results were obtained by reference of experimental peak area ratios to standard curves and data were normalized to tissue weight. Data for each individual lipid were analyzed by ANOVA with Tukey's post hoc test. Error bars are SEM. ${ }^{* *} p<0.01,{ }^{* * *} p<0.001$ compared with untreated; $\# p<0.05$, \#\#p<0.01 compared with NGF.

lites, spinal NGF mechanisms of nociception may be particularly dependent on oxidation of linoleic acid.

\section{NGF-evoked increases in TRPV1-active oxidized lipids in hindpaw skin are attenuated by intraplantar injection of NDGA and BEL}

We next used HPLC/MS to determine whether NGF increased concentrations of oxidized lipid TRPV1 agonists in skin tissues. In hindpaw skin, NGF treatment significantly increased LA (Fig. 6A) and several LA metabolites, including 9-HODE and 13-HODE, 9,10-DiHOME and 12,13-DiHOME, and 12(13)EpOME (Fig. 6B,C). Although AA did not change after NGF treatment (Fig. 6A), NGF increased the AA metabolites 12-HETE and LTB4 (Fig. 6D). Similar to its effects in spinal cord, NDGA injection into plantar skin significantly reduced NGF-evoked elevations in oxidized metabolites (Fig. 6B-D). Consistent with the effects of NDGA, BEL also reduced all NGF-evoked increases in oxidized metabolites, with the exception of 13-oxoODE, 12(13)EpOME, and 12-HETE. Differences in the effects of NDGA and BEL on specific metabolites, as well as effects on LA and AA precursors (Fig. 6A), may reflect different metabolic pathways targeted by these drugs. Although NGF had no effect on AA levels in skin, BEL increased AA concentrations (Fig. 6A) and had differential effects on specific AA metabolites (e.g., 12-HETE; Fig. 6D). Combined with the findings for the peripheral inhibitory effects of BEL, CPZ, and NDGA on NGF-induced thermal hyperalgesia (Fig. 2), these findings support the hypothesis that increased production of oxidized lipid TRPV1 agonists in skin likely contributes to the persistent NGF-induced thermal hyperalgesia.

\section{Discussion}

This study tested the novel hypothesis that NGF triggers the development of a persistent thermal and mechanical nociceptive state mediated by oxidative processes, leading to increased pro- duction of endogenous TRPV1 agonists and activation of the channel. In the present rat model, systemic NGF administration produced physiologically relevant circulating concentrations of NGF (Blandini et al., 2006) accompanied by pain behaviors that persisted beyond detectable circulating levels of the neurotrophin. In addition, the results indicate that TRPV1 mediates these effects of NGF via distinct peripheral and central mechanisms. Importantly, these studies demonstrate a novel role for lipid oxidation in mediating the transition to a persistent pain state through increased production of TRPV1-active lipids in both spinal and cutaneous tissues. Further, the lipidomic analyses reveal that levels of linoleic acid and the OLAMs are $\sim 10$-fold greater in cutaneous tissues compared with spinal cord homogenates. Based on these findings, we propose a novel mechanism for persistent nociception evoked by NGF through increased production of oxidized lipid TRPV1 agonists and subsequent receptor activation at both central and peripheral nerve terminals of sensory neurons.

Consistent with prior studies (Lewin et al., 1993; Hathway and Fitzgerald, 2006; Mills et al., 2013), repeated injection of NGF triggered a persistent nociceptive state that required changes in protein expression and lasted beyond the complete metabolism of the exogenous neurotrophin in circulation. NGF activation of TrkA or p75 receptors on sensory neurons and internalization and trafficking of the neurotrophin-receptor complex to the cell body could initiate changes in gene expression that lead to enhanced sensory transduction or peripheral sensitization (Woolf, 1996; Delcroix et al., 2003). Consistent with this hypothesis, systemic coadministration of the protein synthesis inhibitors anisomycin and cycloheximide with NGF prevented the development of persistent pain behaviors. However, changes in gene expression could also be mediated by the release of additional inflammatory mediators from TrkA or p75 receptor activation in 

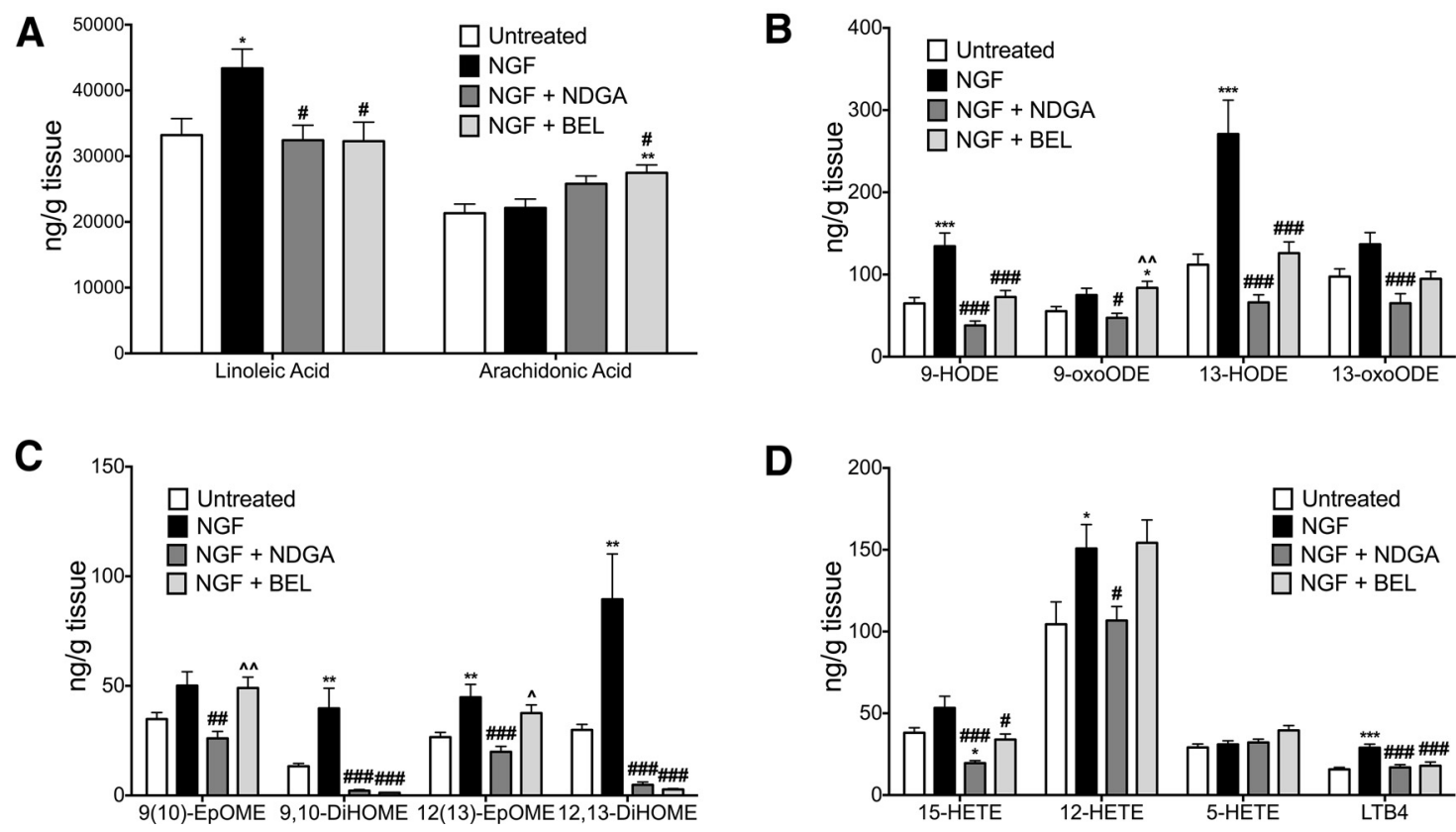

Figure 6. NGF-evoked increases in TRPV1-active oxidized lipids in hindpaw skin were attenuated by intraplantar injection of NDGA and BEL. Hindpaw skin biopsies (6 mm) were rapidly removed from untreated rats, NGF-treated rats, and NGF-treated rats cotreated with NDGA $(5 \mu$ g, i.pl.) or BEL ( $30 \mu$ g, i.pl.) on day $8(n=10-14 /$ group $)$. Lipids were extracted in methanol containing isotope labeled standards for MS analysis of LA and AA (A), HODE and ox0ODE metabolites (B), EpOME and DiHOME metabolites (C), and HETE metabolites and LTB4 in hindpaw skin tissues (D). Quantitative results were obtained by reference of experimental peak area ratios to standard curves and data were normalized to tissue weight. Data for each individual lipid were analyzed by ANOVA with Tukey's post hoc test. Error bars are SEM. ${ }^{*} p<0.05,{ }^{* *} p<0.01,{ }^{* * *} p<0.001$ compared with untreated; $\# p<0.05$, \#\#p $<0.01$ compared with NGF.

non-neuronal cells (Woolf et al., 1996; Ji et al., 2002; Nicol and Vasko, 2007; Mantyh et al., 2011). Further investigation and identification of specific gene changes in neurons evoked by NGF treatment could advance our understanding of the neuronal mechanisms involved in the transition to a persistent pain state. Based on the collective findings in the present study, genes involved in transcription, translation, or posttranslational modification of TRPV1 or in oxidative processes likely contribute to NGF-evoked persistent pain.

The comparison of peripheral versus spinal injection of $\mathrm{CPZ}$ provides insight into mechanisms of persistent NGF nociception. The finding of immediate reversal of persistent NGF nociception with systemic injection of $\mathrm{CPZ}$ demonstrates a pivotal role for TRPV1 in mediating this effect, but does not indicate the site of action. Importantly, the TRPV1 channel is expressed on peripheral and central terminals of certain nociceptive afferent fibers as well as intrinsic second-order neurons in the spinal dorsal horn (Doly et al., 2004; Zhou et al., 2009). The peripheral blockade of thermal hyperalgesia by intraplantar injection of $\mathrm{CPZ}$ is consistent with prior findings that NGF sensitizes thermosensitive afferents (Lewin et al., 1993) and demonstrates that thermal allodynia is initiated by peripheral TRPV1 activation. Based on behavioral, superfusion, biochemical, and electrophysiologic experiments, the present study strongly supports a role of increased peripheral TRPV1 activities in mediating NGF-evoked persistent thermal hyperalgesia. The demonstration of increased capsaicin $E_{\mathrm{MAX}}$ values for $I_{\mathrm{CAP}}$ and CGRP release, together with increased membrane expression of TRPV1 by fractionated Western blot analysis, suggests that NGF treatment evokes sustained accumulation of TRPV1 in the membranes of peripheral afferent terminals and their cell bodies. Because TRPV1 protein quantification in skin was not feasible with WB approaches, it is also possible that posttranslational modifications alone, independent of receptor density, could result in increased capsaicin-evoked inward current and neuropeptide release. Anisomycin and cycloheximide reduced NGF-evoked enhancements in peripheral TRPV1mediated nocifensive responses, consistent with changes in protein translation of TRPV1 and/or genes involved in posttranslational modification or altered surface trafficking of receptor reserves. Persistent thermal hyperalgesia is also mediated by central mechanisms involving TRPV1 activation, because both peripheral and intrathecal injection of $\mathrm{CPZ}$ produced nearcomplete reversal of thermal hyperalgesia. This observation is similar to the effects of intrathecal TRPV1 antagonists in models of inflammation (Cui et al., 2006; Kanai et al., 2007; McGaraughty et al., 2008; Patwardhan et al., 2009). One interpretation of the observation of near-complete efficacy of $\mathrm{CPZ}$ after either intraplantar or intrathecal injection is blockade of TRPV1 on peripheral and central terminals of the same population of afferent neurons. This is consistent with the observation of elevated TRP-active lipids in both skin and spinal cord tissues. Interestingly, fractionated Western blot analysis failed to detect any change in membrane expression of TRPV1 in spinal cord homogenates after NGF treatment. Although these results may reflect a detection issue with the Western blot approach due to the limited amount of TRPV1 protein in the spinal cord, it is also possible that this finding could be due to either selective trafficking of TRPV1 to peripheral terminals of afferent neurons (Ji et al., 2002) or multiple cellular sources of TRPV1 in the dorsal spinal cord (Doly et al., 2004; Zhou et al., 2009), masking the effect of NGF on central terminals of TRPV ${ }^{+}$neurons.

In contrast to thermal hyperalgesia, NGF-evoked mechanical allodynia is mediated by TRPV1 exclusively through spinal mechanisms because intrathecal, but not peripheral, injection of $\mathrm{CPZ}$ reversed this nociceptive state. Other studies using behavioral (Honore et al., 2005; Cui et al., 2006; McGaraughty et al., 2008; Patwardhan et al., 2009) or electrophysiologic (Holzer, 
2008; McGaraughty et al., 2008; Willis, 2009) methods have implicated spinal TRPV1 in mediating mechanical allodynia. The BEL, CPZ, and NDGA experiments, combined with the lipidomic analysis of spinal cord tissues, suggest that NGF triggers an oxidized lipid/TRPV1 pathway leading to spinal cord plasticity and mechanical allodynia. Collectively, our findings indicate that NGF-induced persistent thermal hyperalgesia and mechanical allodynia are mediated by spatially distinct mechanisms that converge through a common oxidative mechanism of increased active production of oxidized TRPV1 agonists.

The behavioral pharmacology and MS studies identify a novel role for oxidative mechanisms in mediating persistent NGF nociception through increased production of oxidized lipid TRPV1 agonists. Peripheral and spinal injections of NDGA, a potent inhibitor of oxidative enzymes and a direct antioxidant (Goodman et al., 1994; DuPont et al., 2001), reversed persistent NGF nociception by a similar magnitude to $\mathrm{CPZ}$, suggesting a link between the actions of both compounds. Furthermore, enzymatic production of oxidized metabolites appears to contribute more to NGF nociception than free radical production because NAC, an antioxidant that reacts directly with free radicals (Nazıroğlu et al., 2013), had no effect on pain behaviors. MS studies provide quantitative evidence to demonstrate that NGF treatment persistently increased TRPV1-active oxidized LA and AA metabolites in hindpaw skin and spinal cord, and increases in oxidized metabolites were reduced by peripheral and central injection of NDGA. Because PLA2 isozymes release free fatty acids from plasma membranes, we investigated whether the PLA2 inhibitor BEL, which has been shown to inhibit both calcium-dependent and calciumindependent cytosolic PLA2 isoforms (Svensson and Yaksh, 2002; Farooqui et al., 2006), alters lipid levels and nociception. Peripheral injection of BEL reduced oxidized lipids and thermal hyperalgesia, but not mechanical allodynia. Conversely, intrathecal administration of BEL reduced oxidized lipid levels and attenuated both thermal hyperalgesia and mechanical allodynia. These results are strikingly similar to those observed with peripheral and spinal CPZ and NDGA and support the hypothesis that PLA2 plays a key role in NGF-evoked generation of TRP-active lipids. However, the present findings do not exclude other possible mechanisms by which spinal PLA2 could modulate nociceptive processing (Svensson and Yaksh, 2002; Lucas et al., 2005; Farooqui et al., 2006). Differences in the effect of NGF on certain metabolites in spinal cord versus hindpaw skin are consistent with reports that TRPV1 modulation and function are tissue specific (Malin et al., 2011) and might also reflect differences in the relative abundance of $\mathrm{AA}$ and $\mathrm{LA}$ in each tissue type. Interestingly, AA was more abundant in spinal cord than LA, and the release of AA by spinal PLA2 is a critical initial step in the generation of spinal COX products associated with inflammatory hyperalgesia (Lucas et al., 2005). In hindpaw skin, LA was more abundant than AA and was significantly increased after NGF treatment. This finding is of particular importance because previous studies have demonstrated that both acute heat (Patwardhan et al., 2010) and postburn injury (Green et al., 2013) generates TRPV1-active OLAMs in skin. The effects of BEL, CPZ, and NDGA on levels of oxidized lipids correspond with the effects of these compounds on pain behaviors, but these compounds are not selective inhibitors of oxidized lipids alone and it is possible that they could reduce persistent nociception through alternative mechanisms.

Collectively, these data demonstrate a novel mechanism for NGF-induced persistent nociception by PLA2 generation of oxidized lipids leading to activation of TRPV1. Because NGF is impli- cated in several chronic pain disorders, increased understanding of oxidative mechanisms mediating the transition to persistent nociception may advance the development of novel analgesics for chronic inflammatory pain.

\section{References}

Aloe L, Tuveri MA, Carcassi U, Levi-Montalcini R (1992) Nerve growth factor in the synovial fluid of patients with chronic arthritis. Arthritis and Rheumatism 35:351-355. CrossRef Medline

Blandini F, Rinaldi L, Tassorelli C, Sances G, Motta M, Samuele A, Fancellu R, Nappi G, Leon A (2006) Peripheral levels of BDNF and NGF in primary headaches. Cephalalgia 26:136-142. CrossRef Medline

Bradford MM (1976) A rapid and sensitive method for the quantitation of microgram quantities of protein utilizing the principle of protein-dye binding. Anal Biochem 72:248-254. CrossRef Medline

Bylund J, Ericsson J, Oliw EH (1998) Analysis of cytochrome P450 metabolites of arachidonic and linoleic acids by liquid chromatography-mass spectrometry with ion trap MS. Anal Biochem 265:55-68. CrossRef Medline

Cattaneo A (2010) Tanezumab, a recombinant humanized mAb against nerve growth factor for the treatment of acute and chronic pain. Curr Opin Mol Ther 12:94-106. Medline

Cui M, Honore P, Zhong C, Gauvin D, Mikusa J, Hernandez G, Chandran P, Gomtsyan A, Brown B, Bayburt EK, Marsh K, Bianchi B, McDonald H, Niforatos W, Neelands TR, Moreland RB, Decker MW, Lee CH, Sullivan JP, Faltynek CR (2006) TRPV1 receptors in the CNS play a key role in broad-spectrum analgesia of TRPV1 antagonists. J Neurosci 26:93859393. CrossRef Medline

Delcroix JD, Valletta JS, Wu C, Hunt SJ, Kowal AS, Mobley WC (2003) NGF signaling in sensory neurons: evidence that early endosomes carry NGF retrograde signals. Neuron 39:69-84. CrossRef Medline

Doly S, Fischer J, Conrath M (2004) The vanilloid receptor-1 (TRPV1) is expressed in some rat dorsal horn NK1 cells. Brain Res 1004:203-207. CrossRef Medline

Dupont R, Goossens JF, Cotelle N, Vrielynck L, Vezin H, Hénichart JP, Cotelle P (2001) New bis-catechols 5-lipoxygenase inhibitors. Bioorganic and Medicinal Chemistry 9:229-235. CrossRef Medline

Dyck PJ, Peroutka S, Rask C, Burton E, Baker MK, Lehman KA, Gillen DA, Hokanson JL, O'Brien PC (1997) Intradermal recombinant human nerve growth factor induces pressure allodynia and lowered heat-pain threshold in humans. Neurology 48:501-505. CrossRef Medline

Fairbanks CA (2003) Spinal delivery of analgesics in experimental models of pain and analgesia. Adv Drug Deliv Rev 55:1007-1041. CrossRef Medline

Farooqui AA, Ong WY, Horrocks LA (2006) Inhibitors of brain phospholipase A2 activity: their neuropharmacological effects and therapeutic importance for the treatment of neurologic disorders. Pharmacol Rev 58: 591-620. CrossRef Medline

Gibbs JL, Flores CM, Hargreaves KM (2006) Attenuation of capsaicinevoked mechanical allodynia by peripheral neuropeptide Y Y1 receptors. Pain 124:167-174. CrossRef Medline

Goodman Y, Steiner MR, Steiner SM, Mattson MP (1994) Nordihydroguaiaretic acid protects hippocampal neurons against amyloid beta-peptide toxicity, and attenuates free radical and calcium accumulation. Brain Res 654:171-176. CrossRef Medline

Green DP, Ruparel S, Roman L, Henry MA, Hargreaves KM (2013) Role of endogenous TRPV1 agonists in a postburn pain model of partialthickness injury. Pain 154:2512-2520. CrossRef Medline

Gregus AM, Doolen S, Dumlao DS, Buczynski MW, Takasusuki T, Fitzsimmons BL, Hua XY, Taylor BK, Dennis EA, Yaksh TL (2012) Spinal 12lipoxygenase-derived hepoxilin A3 contributes to inflammatory hyperalgesia via activation of TRPV1 and TRPA1 receptors. Proc Natl Acad Sci U S A 109:6721-6726. CrossRef Medline

Hargreaves K, Dubner R, Brown F, Flores C, Joris J (1988) A new and sensitive method for measuring thermal nociception in cutaneous hyperalgesia. Pain 32:77-88. CrossRef Medline

Hathway GJ, Fitzgerald M (2006) Time course and dose-dependence of nerve growth factor-induced secondary hyperalgesia in the mouse. J Pain 7:57-61. Medline

Holzer P (2008) The pharmacological challenge to tame the transient receptor potential vanilloid-1 (TRPV1) nocisensor. Br J Pharmacol 155:11451162. CrossRef Medline

Honore P, Wismer CT, Mikusa J, Zhu CZ, Zhong C, Gauvin DM, Gomtsyan A, El 
Kouhen R, Lee CH, Marsh K, Sullivan JP, Faltynek CR, Jarvis MF (2005) A-425619 [1-isoquinolin-5-yl-3-(4-trifluoromethyl-benzyl)-urea], a novel transient receptor potential type V1 receptor antagonist, relieves pathophysiological pain associated with inflammation and tissue injury in rats. J Pharmacol Exp Ther 314:410-421. CrossRef Medline

Hwang SW, Cho H, Kwak J, Lee SY, Kang CJ, Jung J, Cho S, Min KH, Suh YG, Kim D, Oh U (2000) Direct activation of capsaicin receptors by products of lipoxygenases: endogenous capsaicin-like substances. Proc Natl Acad Sci U S A 97:6155-6160. CrossRef Medline

Iannone F, De Bari C, Dell'Accio F, Covelli M, Patella V, Lo Bianco G, Lapadula G (2002) Increased expression of nerve growth factor (NGF) and high affinity NGF receptor (p140 TrkA) in human osteoarthritic chondrocytes. Rheumatology (Oxford) 41:1413-1418. CrossRef Medline

Ji RR, Samad TA, Jin SX, Schmoll R, Woolf CJ (2002) p38 MAPK activation by NGF in primary sensory neurons after inflammation increases TRPV1 levels and maintains heat hyperalgesia. Neuron 36:57-68. CrossRef Medline

Kanai Y, Hara T, Imai A, Sakakibara A (2007) Differential involvement of TRPV1 receptors at the central and peripheral nerves in CFA-induced mechanical and thermal hyperalgesia. J Pharm Pharmacol 59:733-738. CrossRef Medline

Konkel A, Schunck WH (2011) Role of cytochrome P450 enzymes in the bioactivation of polyunsaturated fatty acids. Biochim Biophys Acta 1814: 210-222. CrossRef Medline

Lewin GR, Ritter AM, Mendell LM (1993) Nerve growth factor-induced hyperalgesia in the neonatal and adult rat. J Neurosci 13:2136-2148. Medline

Lewin GR, Rueff A, Mendell LM (1994) Peripheral and central mechanisms of NGF-induced hyperalgesia. Eur J Neurosci 6:1903-1912. CrossRef Medline

Lucas KK, Svensson CI, Hua XY, Yaksh TL, Dennis EA (2005) Spinal phospholipase A2 in inflammatory hyperalgesia: role of group IVA cPLA2. Br J Pharmacol 144:940-952. CrossRef Medline

Malin S, Molliver D, Christianson JA, Schwartz ES, Cornuet P, Albers KM, Davis BM (2011) TRPV1 and TRPA1 function and modulation are target tissue dependent. J Neurosci 31:10516-10528. CrossRef Medline

Mantyh PW, Koltzenburg M, Mendell LM, Tive L, Shelton DL (2011) Antagonism of nerve growth factor-TrkA signaling and the relief of pain. Anesthesiology 115:189-204. CrossRef Medline

McGaraughty S, Chu KL, Brown BS, Zhu CZ, Zhong C, Joshi SK, Honore P, Faltynek CR, Jarvis MF (2008) Contributions of central and peripheral TRPV1 receptors to mechanically evoked and spontaneous firing of spinal neurons in inflamed rats. J Neurophysiol 100:3158-3166. CrossRef Medline

Mills CD, Nguyen T, Tanga FY, Zhong C, Gauvin DM, Mikusa J, Gomez EJ, Salyers AK, Bannon AW (2013) Characterization of nerve growth factor-induced mechanical and thermal hypersensitivity in rats. Eur J Pain 17:469-479. CrossRef Medline

Nazıroğlu M, Ciğ B, Ozgül C (2013) Neuroprotection induced by $\mathrm{N}$-acetylcysteine against cytosolic glutathione depletion-induced $\mathrm{Ca} 2+$ influx in dorsal root ganglion neurons of mice: role of TRPV1 channels. Neuroscience 242:151-160. CrossRef Medline

Nicol GD, Vasko MR (2007) Unraveling the story of NGF-mediated sensitization of nociceptive sensory neurons: ON or OFF the Trks? Mol Interv 7:26-41. CrossRef Medline

Paterson S, Schmelz M, McGlone F, Turner G, Rukwied R (2009) Facilitated neurotrophin release in sensitized human skin. Eur J Pain 13:399-405. CrossRef Medline

Patwardhan AM, Scotland PE, Akopian AN, Hargreaves KM (2009) Activation of TRPV1 in the spinal cord by oxidized linoleic acid metabolites contributes to inflammatory hyperalgesia. Proc Natl Acad Sci U S A 106: 18820-18824. CrossRef Medline

Patwardhan AM, Akopian AN, Ruparel NB, Diogenes A, Weintraub ST, Uhlson C, Murphy RC, Hargreaves KM (2010) Heat generates oxidized li- noleic acid metabolites that activate TRPV1 and produce pain in rodents. J Clin Invest 120:1617-1626. CrossRef Medline

Petty BG, Cornblath DR, Adornato BT, Chaudhry V, Flexner C, Wachsman M, Sinicropi D, Burton LE, Peroutka SJ (1994) The effect of systemically administered recombinant human nerve growth factor in healthy human subjects. Ann Neurol 36:244-246. CrossRef Medline

Rukwied R, Mayer A, Kluschina O, Obreja O, Schley M, Schmelz M (2010) NGF induces non-inflammatory localized and lasting mechanical and thermal hypersensitivity in human skin. Pain 148:407-413. CrossRef Medline

Ruparel NB, Patwardhan AM, Akopian AN, Hargreaves KM (2008) Homologous and heterologous desensitization of capsaicin and mustard oil responses utilize different cellular pathways in nociceptors. Pain 135:271279. CrossRef Medline

Ruparel S, Green D, Chen P, Hargreaves KM (2012a) The cytochrome P450 inhibitor, ketoconazole, inhibits oxidized linoleic acid metabolitemediated peripheral inflammatory pain. Mol Pain 8:73. CrossRef Medline

Ruparel S, Henry MA, Akopian A, Patil M, Zeldin DC, Roman L, Hargreaves KM (2012b) Plasticity of cytochrome P450 isozyme expression in rat trigeminal ganglia neurons during inflammation. Pain 153:2031-2039. CrossRef Medline

Sarchielli P, Mancini ML, Floridi A, Coppola F, Rossi C, Nardi K, Acciarresi M, Pini LA, Calabresi P (2007) Increased levels of neurotrophins are not specific for chronic migraine: evidence from primary fibromyalgia syndrome. J Pain 8:737-745. CrossRef Medline

Svensson CI, Yaksh TL (2002) The spinal phospholipase-cyclooxygenaseprostanoid cascade in nociceptive processing. Annu Rev Pharmacol Toxicol 42:553-583. CrossRef Medline

Svensson P, Cairns BE, Wang K, Arendt-Nielsen L (2003) Injection of nerve growth factor into human masseter muscle evokes long-lasting mechanical allodynia and hyperalgesia. Pain 104:241-247. CrossRef Medline

Tria MA, Fusco M, Vantini G, Mariot R (1994) Pharmacokinetics of nerve growth factor (NGF) following different routes of administration to adult rats. Exp Neurol 127:178-183. CrossRef Medline

Wen H, Östman J, Bubb KJ, Panayiotou C, Priestley JV, Baker MD, Ahluwalia A (2012) 20-Hydroxyeicosatetraenoic acid (20-HETE) is a novel activator of transient receptor potential vanilloid 1 (TRPV1) channel. J Biol Chem 287:13868-13876. CrossRef Medline

Willis WD Jr (2009) The role of TRPV1 receptors in pain evoked by noxious thermal and chemical stimuli. Exp Brain Res 196:5-11. CrossRef Medline

Woolf CJ (1996) Phenotypic modification of primary sensory neurons: the role of nerve growth factor in the production of persistent pain. Philosophical Transactions of the Royal Society of London Series B, Biological Sciences 351:441-448. CrossRef Medline

Woolf CJ, Ma QP, Allchorne A, Poole S (1996) Peripheral cell types contributing to the hyperalgesic action of nerve growth factor in inflammation. J Neurosci 16:2716-2723. Medline

Yaksh TL, Farb DH, Leeman SE, Jessell TM (1979) Intrathecal capsaicin depletes substance $\mathrm{P}$ in the rat spinal cord and produces prolonged thermal analgesia. Science 206:481-483. CrossRef Medline

Zhou HY, Chen SR, Chen H, Pan HL (2009) The glutamatergic nature of TRPV1-expressing neurons in the spinal dorsal horn. J Neurochem 108: 305-318. CrossRef Medline

Zhu W, Oxford GS (2007) Phosphoinositide-3-kinase and mitogen activated protein kinase signaling pathways mediate acute NGF sensitization of TRPV1. Mol Cell Neurosci 34:689-700. CrossRef Medline

Zhu W, Oxford GS (2011) Differential gene expression of neonatal and adult DRG neurons correlates with the differential sensitization of TRPV1 responses to nerve growth factor. Neurosci Lett 500:192-196. CrossRef Medline

Zhu W, Galoyan SM, Petruska JC, Oxford GS, Mendell LM (2004) A developmental switch in acute sensitization of small dorsal root ganglion (DRG) neurons to capsaicin or noxious heating by NGF. J Neurophysiol 92:3148-3152. CrossRef Medline 